GLOBAL EXISTENCE OF WEAK SOLUTIONS

FOR INTERFACE EQUATIONS COUPLED WITH DIFFUSION EQUATIONS

\author{
By \\ Yoshikazu Giga \\ Shun'ichi Goto \\ and
}

Hitoshi Ishii

IMA Preprint Series \# 806

May 1991 


\title{
GLOBAL EXISTENCE OF WEAK SOLUTIONS FOR INTERFACE EQUATIONS COUPLED WITH DIFFUSION EQUATIONS
}

\author{
YOSHIKAZU GIGA*, SHUN'ICHI GOTO** AND HITOSHI ISHII $†$
}

\begin{abstract}
A weak formulation for an interface dynamics coupled with a diffusion equation is introduced. A global-in-time weak solution is constructed for an arbitrary initial data under a periodic boundary condition. The result applies to the interface equation obtained as a certain singular limit of some reaction-diffusion systems including the activator-inhibitor model.
\end{abstract}

Key words. Interface equation with diffusion equation, global existence, viscosity solution

AMS(MOS) subject classifications. 35K55, (35K57 35K65)

1. Introduction. This paper is concerned with interface equations coupled with diffusion equations. A typical example is formally obtained as a certain singular limit of a class of reaction diffusion systems [XYC]. Our main objective is to construct a global-in-time weak solution for the initial value problem of these interface equations.

Let $\Omega_{ \pm}(t)$ be two disjoint open sets in $\mathbb{R}^{n}$ depending on time $t$. The complement of the union of $\Omega_{+}(t)$ and $\Omega_{-}(t)$ is called the interface and denoted by $\Gamma(t)$. To write down the equation we assume that the interface $\Gamma(t)$ is a smooth hypersurface so that $\Gamma(t)$ is the boundary of $\Omega_{ \pm}(t)$. Let $V=V(t, x)$ denote the speed of $\Gamma(t)$ at $x \in \Gamma(t)$ in the normal direction $\vec{n}$ from $\Omega_{+}(t)$ to $\Omega_{-}(t)$. Let $\kappa(=\operatorname{div} \vec{n})$ denote $(n-1$ times) the mean curvature of $\Gamma(t)$ at $x \in \Gamma(t)$. We consider an interface equation for $\Gamma(t)$ :

$$
V=W(v)-c \kappa \quad \text { on } \Gamma(t)
$$

coupled with a diffusion equation for $v=v(t, x)$ :

$$
v_{t}=D \Delta v+g_{ \pm}(v), x \in \Omega_{ \pm}(t), t>0,
$$

where $c \geq 0$ and $D>0$ are constants. Here $g_{ \pm}$and $W$ are given bounded continuous functions on $\mathbb{R}$. We also impose a condition that $v(t)=v(t, \cdot)$ is continuous in $\mathbb{R}^{n}$ with its first derivatives, i.e.,

$$
v(t)=v(t, \cdot) \in C^{1}\left(\mathbb{R}^{n}\right) \quad \text { for } t>0 .
$$

Our goal is to construct a global solution of the initial value problem for interface equations coupled with diffusion equations - a typical example of which is (1.1)-(1.3). There is an intrinsic difficulty to construct a global solution $\left(\Omega_{ \pm}(t), v(t)\right)_{t>0}$ since $\Gamma(t)$ may have singularities in a finite time. If $v$ is a constant so that $g_{ \pm}(v)=0,(1.1)-(1.3)$ becomes

$$
V=C-c \kappa,
$$

*Department of Mathematics, Hokkaido University, Sapporo 060, JAPAN. The work of this author was partially supported by the Grant-in-Aid for Scientific Research 01740076, 01540092, The Japan Ministry of Education, Science and Culture. JAPAN.

**Department of Applied Science, Faculty of Engineering, Kyushu University 36, Fukuoka 812,

†Department of Mathematics, Chuo University, Bunkyo-ku, Tokyo 112, Japan. The work of this author was partially supported by the Grant-in-Aid for Scientific Research 02640150, the Japan Ministry of Education, Science and Culture. 
where $C$ is a constant. If $C=0$ and $c>0,(1.4)$ becomes

$$
V=-c \kappa,
$$

which is called the mean curvature flow equation. Even for (1.5) Grayson [Gr] gives an example of a barbell in $\mathbb{R}^{3}$ with a long, thin handle that actually pinches off in a finite time. To track whole evolution of interface we interpret $\Gamma(t)$ as a level set of viscosity solution of some second order evolution equations as in [CGG]. In fact Y.-G. Chen and the first two authors [CGG] constructed a unique global weak solution with arbitrary initial data for a class of interface equations including (1.4) and (1.1) where $v$ only depends on time; see [GG1] for interface equations that the theory in [CGG] applies to. Almost at the same time Evans and Spruck [ES1] constructed the same solution but only for (1.5). Another formulation closely related to [CGG] and [ES1] is given in [S]. We refer to [ES2] and [GG2] for further development of the theory and references. We note that idea using level of viscosity solutions for $V=C$ is also found in an unpublished paper of Barles [B].

Although the interface equation admits a global weak solution, $\Gamma(t)$ may develop an interior (Remark 2.5). We introduce a generalized formulation of (1.2)-(1.3). For technical reasons we impose a periodic boundary condition. In this paper among other results we construct a global weak solution of the initial value problem for (1.1)-(1.3) with arbitrary initial data $(\Gamma(0), v(0, x)), v(0, x) \in C^{2}$ under the periodic boundary condition (Theorem 4.6). (We may assume $D=1$ without the loss of generality). For this purpose for continuous $v$ we construct a unique global weak solution for (1.1). The basic idea is the same as in [CGG] but we are forced to use results in [GGIS] since $v$ may depend on $x$ as well as $t$. We solve (1.2) - (1.3) with above $v$ and $\Omega_{ \pm}(t)$ determined by (1.1) with the initial condition. If we write the solution by $w$ we have a mapping $v \mapsto w$. Since our weak formulation forces us to interpret the mapping $v \mapsto w$ as a multi-valued mapping, we use Kakutani (-Ky Fan's) fixed point theory (see e.g. $[\mathrm{AF}]$ ) to get a global generalized solution as a fixed point of the mapping $v \mapsto w$. Our results applies to the system (1.2) - (1.3) with (1.1) replaced by more general interface equations including anisotropic motion ( $\operatorname{cf}[\mathrm{Gu} 1,2],[\mathrm{C}])$. We do not know the uniqueness of our solutions.

Let us mention some results on (1.1) - (1.3) related to ours. In [XYC] X.-Y. Chen constructed a unique local smooth solution for a smooth initial data $(\Gamma(0), v(0, x))$ in $\mathbb{R}^{n}$ when $c>0$. When $n=1$, the curvature term in (1.1) disappears. Hilhorst, Nishiura and Mimura [HNM] constructed a global unique solution for (1.1) - (1.3) when the interface is a point and $n=1$ under the Neumann boundary condition. Their interface is $C^{1}$ in time. After this work was completed, we learned of the recent paper of $\mathrm{X}$. Chen [XC2] which extends the local existence results in $[\mathrm{XYC}]$ to the case $c=0$. Our result seems to be a first global result even for (1.1) $-(1.3)$ with $c>0$ or $c=0$ when $n>1$.

Interface equations and reaction-diffusion equations are closely related; see $[\mathrm{F}]$. Typical examples of the system (1.1) - (1.3) is provided as a singular limit of reactiondiffusion equations at least formally; see $[\mathrm{OMK}]$ and $[\mathrm{XYC}]$. We will explain it more explicitly following $[\mathrm{XYC}]$. We consider a reaction-diffusion system describing the activator-inhibitor model:

$$
\begin{array}{ll}
u_{t}=\varepsilon \Delta u+\frac{1}{\varepsilon} f(u, v), & x \in \mathbb{R}^{n}, t>0, \\
v_{t}=D \Delta v+g(u, v), & x \in \mathbb{R}^{n}, t>0,
\end{array}
$$


with

$$
\begin{aligned}
& f(u, v)=f_{0}(u)-v, f_{0}(u)=u(1-u)(u-a), \\
& g(u, v)=u-\gamma v
\end{aligned}
$$

where $\gamma>0,0<a<1$ and $\varepsilon$ is a small positive parameter. The zero set of $f$ consists of three branches

$$
\begin{array}{ll}
u=h_{-}(v) & \text { for } u<a_{-} \\
u=h_{+}(v) & \text { for } a_{+}<u \\
u=h_{0}(v) & \text { for } a_{-}<u<a_{+}
\end{array}
$$

where $a_{-}<a_{+}$and $f_{0}^{\prime}\left(a_{-}\right)=f_{0}^{\prime}\left(a_{+}\right)=0$. When $\varepsilon \rightarrow 0$, it is expected that $u$ tends to $h_{ \pm}(v)$ in some region $\Omega_{ \pm}(t)$ in $\mathbb{R}^{n}$ since $h_{ \pm}(v)$ is a stable zero of $u_{t}=f(u, v)$. From (1.6) it is also expected that the interface $\Gamma(t)$ moves by (1.1) with $c=\varepsilon$. Here $W(b)$ for $b, f_{0}\left(a_{-}\right)<b<f_{0}\left(a_{+}\right)$is the speed of the travelling wave of

$$
u_{t}=\Delta u+f(u, b)
$$

and is given by

$$
W(b)=\frac{1}{\sqrt{2}}\left(h_{+}(b)+h_{-}(b)-h_{0}(b)\right) ;
$$

see Aronson and Weinberger [AW]. The equation (1.7) now becomes (1.2) as $\varepsilon \rightarrow 0$ by taking $g_{ \pm}(v)=g\left(h_{ \pm}(v), v\right)$. For more details we refer to [OMK] and [XYC] and references theirin. We note that anistropic interface equations are also derived by a singular limit of some reaction-diffusion equation [C].

There is an extensive literature on the behavior $u^{\varepsilon}$ as $\varepsilon \downarrow 0$ in (1.6) and its relation to the solutions of interface equation when $v$ is given and the space dimension $n=1$. See e.g. [FH], [BK], [CP]. Recently, some results are extended to the case $n>1$ where the curvature effect comes in. If $v$ is a constant and $W(v)=0,(1.6)$ is called the AllenCahn equation whose relation to (1.5) with $c>0$ is rigorously analyzed by Bronsard and Kohn [BK] and DeMottoni and Schatzman [DS]. X. Chen [XC1] extended results of [DS] and simplified the argument. After this work was completed, we learned that $\mathrm{X}$. Chen [XC2] derived (1.1) - (1.3) with $c=0$ rigorously as a singular limit of (1.6) (1.7). There is also an argument to interpret the case $c=\varepsilon>0$ in [XC2]. His method is an extension of his work [XC1]. All results in [BK, DS, XC1, XC2] assume that the solution of the interface equation is smooth to get the behavior of $u^{\varepsilon}$ as $\varepsilon \downarrow 0$. Very recently we learned that Evans, Soner and Songanidis [ESS] obtained the behavior of $u^{\varepsilon}$ even after singularities appear on the interface for the Allen-Cahn equation.

In $\S 2$ we solve a general interface equation including (1.1) for given continuous function $v$ globally in time under a periodic boundary condition. In $\S 3$ we give a generalized formulation of $(1.2)-(1.3)$. In $\S 4$ we state our main existence results and prove them by a fixed point argument. In Appendix we state a stability property of viscosity solutions used in $\S 4$.

After this work was completed, X.-Y. Chen kindly informed that he found another proof for global existence for (4.1), (4.2), $\left(4.3^{\prime}\right)$ with $c>0$ without using a fixed point argument for multi-valued mappings. 
2. Interface equations. We consider interface equations under periodic boundary conditions. The periodic boundary condition is important because it is often used in numerical experiments. For $\alpha_{i}>0(1 \leq i \leq n)$ let $R$ be a rectangle in $\mathbb{R}^{n}$ of the form

$$
R=\left\{\left(x_{1}, \cdots, x_{n}\right) \in \mathbb{R}^{n} ; 0 \leq x_{i} \leq \alpha_{i}, 1 \leq i \leq n\right\} .
$$

We identify faces $x_{i}=0$ and $x_{i}=\alpha_{i}(1 \leq i \leq n)$ of $R$ to obtain an $n$-dimensional flat torus $\mathbf{T}$. Motion of interfaces in $R$ under periodic boundary conditions is interpreted as the motion in $\mathbb{T}$. We consider $\mathbf{T}$ rather than $\mathbb{R}^{n}$ for later technical convenience because $T$ is compact and has no boundary.

Let $\Omega_{ \pm}(t)$ be an open set in $T$ depending on time $t \geq 0$ such that $\Omega_{+}(t) \cap \Omega_{-}(t)=$ $\phi$. Let $\Gamma(t)$ denote the complement of $\Omega_{+}(t) \cup \Omega_{-}(t)$ in $\mathbf{T}$. Physically speaking, $\Gamma(t)$ is called an interface bounding two phases $\Omega_{ \pm}(t)$ of material, e.g. solid and liquid region. Suppose that $\Gamma(t)$ is a smooth hypersurface and let $\vec{n}$ denote the unit normal vector field pointing from $\Omega_{+}(t)$ to $\Omega_{-}(t)$. Let $V=V(t, x)$ denote the speed of $\Gamma(t)$ at $x \in \Gamma(t)$ in the direction $\vec{n}$. It is convenient to extend $\vec{n}$ to a vector field (still denoted by $\vec{n}$ ) on a tubular neighborhood of $\Gamma(t)$ such that $\vec{n}$ is constant in the normal direction of $\Gamma(t)$. The equation for $\Gamma(t)$ we consider here is of the form

$$
\begin{aligned}
V & =\xi(t, x, \vec{n}, \nabla \vec{n}) \\
& :=\eta(\vec{n}, \nabla \vec{n})+\omega(t, x, \vec{n}) \text { on } \Gamma(t),
\end{aligned}
$$

where $\eta$ and $\omega$ are given functions and $\nabla$ stands for the spatial gradient in $\mathbb{T}$. A typical example is

$$
V=c \operatorname{div} \vec{n}+\omega(t, x),
$$

where $c$ is a nonnegative constant and $\omega$ is independent of $\vec{n}$. The equation (2.2) is called the mean curvature flow equation if $c>0$ and $\omega \equiv 0$. A reason we consider general (2.1) is to include anisotropic motion as in [Gu1,2].

We next introduce a weak formulation for (2.1) following [CGG, GG1]. For $\eta$ we set

$$
\begin{aligned}
F_{\eta}(p, X): & =-|p| \eta\left(-\bar{p},-Q_{\bar{p}}(X)\right), \bar{p}=p /|p| \\
Q_{\bar{p}}(X) & =R_{\bar{p}} X R_{\bar{p}} \text { with } R_{\bar{p}}=I-\bar{p} \otimes \bar{p}
\end{aligned}
$$

where $p \in \mathbb{R}^{n} \backslash\{0\}$ and $X \in \mathbb{S}_{n}$, the space of $n \times n$ real symmetric matrices. We also set

$$
F_{\xi}(t, x, p, X):=F_{\eta}(p, X)-\omega(t, x,-\bar{p})|p| .
$$

For example a calculation shows

$$
F_{\eta}(p, X)=-c \operatorname{trace}((I-\bar{p} \otimes \bar{p}) X)
$$

if

$$
\eta(\vec{n}, \nabla \vec{n})=-c \operatorname{div} \vec{n}
$$

as in (2.2). The following definition of weak solutions for (2.1) is a variant of that in [CGG, GG1]. For the definition of (viscosity) sub- and supersolutions and viscosity solutions; see e.g. [GGIS]. 
Definition 2.1. Let $\left\{\Omega_{ \pm}(t)\right\}_{0<t<T}$ be a one parameter family of open sets in T such that $\Omega_{+}(t) \cap \Omega_{-}(t)=\phi$. Suppose that there is a viscosity solution $u \in$ $C([0, T) \times \mathbb{T})$ of

$$
u_{t}+F_{\xi}\left(t, x, \nabla u, \nabla^{2} u\right)=0 \text { in }(0, T) \times \mathbb{T}
$$

such that

$$
\Omega_{ \pm}(t)=\left\{x \in \mathbb{R}^{n} ; u(t, x) \gtrless 0\right\} \text { for } 0 \leq t<T .
$$

We say $\left\{\Omega_{ \pm}(t)\right\}_{0 \leq t<T}$ is a weak solution of $(2.1)$ in $(0, T)$ with initial data $\Omega_{ \pm}(0)$. Here $F_{\xi}$ is defined by $(2.3)-(2.4)$.

Roughly speaking, if (2.1) is parabolic (not necessarily strictly parabolic), $\eta$ grows linearly in $\nabla \vec{n}$ then one can claim the unique global existence of weak solutions for (2.1) with given initial data $\Omega_{ \pm}(0)$ provided that $\eta$ and $\omega$ are continuous. If $\omega$ is independent of $x$ and $T$ is replaced by $\mathbb{R}^{n}$, the unique global existence is now well known if one of $\Omega_{ \pm}(0)$ is bounded (cf. [CGG, GG1]). We now list our assumptions on $\eta$ and $\omega$.

$\eta$ is a real valued continuous function on the vector bundle

$$
\begin{aligned}
E=\left\{\left(\bar{p}, Q_{\bar{p}}(X)\right) ; \bar{p} \in S^{n-1}, X \in \mathbb{S}_{n}\right\} \\
\\
\text { over a unit sphere } S^{n-1} .
\end{aligned}
$$

$$
\begin{gathered}
\eta\left(-\bar{p},-Q_{\bar{p}}(X)\right) \geq \eta\left(-\bar{p},-Q_{\bar{p}}(Y)\right) \text { for } X \geq Y, \bar{p} \in S^{n-1}, \\
\text { where } \mathbb{S}_{n} \text { is equipped with usual ordering } .
\end{gathered}
$$

$$
\begin{aligned}
& \liminf _{\rho \downarrow 0} \rho \inf _{|\bar{p}|=1} \eta\left(-\bar{p}, \frac{I-\bar{p} \otimes \bar{p}}{\rho}\right)>-\infty \\
& \quad \limsup _{\rho \downarrow 0} \rho \sup _{|\bar{p}|=1} \eta\left(-\bar{p}, \frac{-I+\bar{p} \otimes \bar{p}}{\rho}\right)<\infty .
\end{aligned}
$$

All assumptions on $\eta$ is found in [GG1]; (2.10) means that $-\eta$ is degenerate elliptic and (2.11) restricts the growth of $\eta$ in $\nabla \vec{n}$. The only assumption for $\omega$ is (2.12)

Theorem 2.2. Assume (2.9) - (2.12) for $\eta$ and $\omega$. Let $\Omega_{ \pm}(0)$ be mutually disjoint open sets in $\mathbf{T}$. Then there is a unique global weak solution $\left\{\Omega_{ \pm}(t)\right\}_{0 \leq t<T}$ of (2.1) in $(0, T)$ with initial data $\Omega_{ \pm}(0)$. (The case $T=\infty$ is included.)

The basic idea of the proof is the same as [CGG, Theorems 6.8, 7.1]; see also [GG1] for relation between assumptions on $\eta$ and $F_{\eta}$. The major technical difference is that the comparison theorem in [CGG] does not apply to (2.7) because $F_{\xi}$ depends on $x$. Instead we apply [GGIS, Theorem 4.1] to get a comparison principle for (2.7). For the reader's convenience we state a version of the comparison principle which follows from [GGIS, Theorem 4.1] and give a brief proof of Theorem 2.2. 
Proposition 2.3. Assume (2.9) - (2.12). Let $u$ and $v$ be, respectively, (viscosity) sub- and supersolutions of (2.7). Assume that $u$ and $v$ are, respectively, upper and lower semicontinuous functions on $[0, T) \times \mathrm{T}$. If

$$
u(0, x) \leq v(0, x) \text { on } \mathbf{T}
$$

then $u(t, x) \leq v(t, x)$ on $[0, T) \times \mathbb{T}$.

Proof. To apply [GGIS, Theorem 4.1] we extend $u, v$ and $\omega$ periodically in space variables outside $R$ and regard (2.7) as

$$
u_{t}+F_{\xi}\left(t, x, \nabla u, \nabla^{2} u\right)=0 \text { in }\left(0, T^{\prime}\right] \times \mathbb{R}^{n},
$$

where $T^{\prime}$ is an arbitrary positive number less than $T$.

We first check assumptions of equation in [GGIS]. By (2.9) - (2.10) we know $F_{\eta}$ satisfies all assumptions on $F$ in [GGIS, Theorem 4.1]. Except the boundedness of $F_{\eta}(p, X)$ on a bounded set in $\left(\mathbb{R}^{n} \backslash\{0\}\right) \times \mathbb{S}_{n}$ the proof is found in [GG1]. This boundedness of $F_{\eta}$ can be proved similarly to the proof of [GG1, Lemma 3.5].

By $(2.12)$ we see $\omega$ is uniformly continuous in $\left[0, T^{\prime}\right] \times \mathbb{R}^{n} \times S^{n-1}$ so $F_{\xi}$ satisfies the uniform continuity assumption in $x$ of [GGIS, (F8)]: there is a modulus $\sigma$ (i.e. $\sigma:[0, \infty) \rightarrow[0, \infty)$ is continuous, nondecreasing and $\sigma(0)=0)$ such that

$$
\left|F_{\xi}(t, x, p, X)-F_{\xi}(t, y, p, X)\right| \leq \sigma(|x-y|(|p|+1))
$$

for $x, y \in \mathbb{R}^{n}, t \in\left[0, T^{\prime}\right], p \in \mathbb{R}^{n} \backslash\{0\}, X \in \mathbb{S}_{n}$. All other assumptions on $F$ in [GGIS, Theorem 4.1] are fulfilled since $\omega$ satisfies (2.12) and $F_{\eta}$ satisfies all assumptions on $F$.

Since $u$ and $v$ is extended periodically and $R$ is bounded, it is not difficult to see that $u$ and $v$ satisfy all the assumptions of [GGIS, Theorem 4.1].

We now apply [GGIS, Theorem 4.1] to conclude $u \leq v$ on $\left[0, T^{\prime}\right] \times \mathbb{R}^{n}$. Since $T^{\prime}<T$ is arbitrary, this completes the proof.

Proof of Theorem 2.2. (Uniqueness). Suppose that $u, v \in C([0, T) \times \mathbb{T})$ solves (2.7) such that

$$
\Omega_{ \pm}(0)=\{x \in \mathbb{T}, u(0, x) \gtrless 0\}=\{x \in \mathbb{T} ; v(0, x) \gtrless 0\} .
$$

By [CGG, Lemma 7.2] there is a continuous nondecreasing function $\theta: \mathbb{R} \rightarrow \mathbb{R}$ with $\theta(0)=0$ such that

$$
u(0, x) \leq \theta(v(0, x))
$$

Since $F_{\xi}$ is geometric, i.e.,

$$
\begin{gathered}
F_{\xi}(t, x, \lambda p, \lambda X+\sigma p \otimes p)=\lambda F_{\xi}(t, x, p, X) \\
\text { for } \lambda>0, \sigma \in \mathbb{R}, t \in(0, T), x \in \mathbf{T}, p \in \mathbb{R}^{n} \backslash\{0\}, X \in \mathbb{S}_{n},
\end{gathered}
$$

by [CGG, Theorem 5.2] we see $\theta(v(t, x))$ also solves (2.7). From Proposition 2.3 it follows $u \leq \theta(v)$ on $[0, T) \times \mathbf{T}$. We thus observe that $u>0$ implies $v>0$ and $v<0$ implies $u<0$. A parallel argument yields the converse implication so $\Omega_{ \pm}(t)$ is determined by $\Omega_{ \pm}(0)$ and is independent of the choice of $u$. This proves the uniqueness of weak solutions. 
(Existence). For given $\Omega_{ \pm}(0)$ we take $u_{0}(x) \in C(\mathbf{T})$ such that

$$
\Omega_{ \pm}(0)=\left\{x \in \mathbf{T} ; u_{0}(x) \gtrless 0\right\} .
$$

Since (2.11) is assumed, one may apply [CGG, Proposition 6.4] to $(2.7)$ in $\left[0, T^{\prime}\right] \times \mathbb{R}^{n}$ with periodic initial data and find sub- and supersolutions $v_{-}, v_{+}$of $(2.7)$ on $\left[0, T^{\prime}\right] \times \mathbb{R}^{n}$ such that

$$
\begin{gathered}
v_{ \pm}(0, x)=u_{0}(x) \text { on } \mathbb{R}^{n} \\
v_{-}(t, x) \leq u_{0}(x) \leq v_{+}(t, x) \text { on }\left[0, T^{\prime}\right] \times \mathbb{R}^{n},
\end{gathered}
$$

where $T^{\prime}<T$. The dependence of $x$ in $F_{\xi}$ is allowed in [CGG, Proposition 6.4]. A trivial modification of the argument enables us to take $v_{-}, v_{+}$as functions on $\left[0, T^{\prime}\right] \times \mathbf{T}$.

Existence of $v_{ \pm}$yields a viscosity solution $u \in C\left(\left[0, T^{\prime}\right] \times \mathbb{T}\right)$ of $(2.7)$ with $u(0, x)=$ $u_{0}(x)$ by Perron's method and Proposition 2.3. Since $T^{\prime}<T$ is arbitrary and the solution is unique, we now obtain a weak solution $\left\{\Omega_{ \pm}(t)\right\}_{0 \leq t<T}$ for initial data $\Omega_{ \pm}(0)$.

Note that the scaling property $(2.13)$ also used to construct $v_{ \pm}$. $\square$

Remark 2.4. The family $\left\{\Omega_{+}(t)\right\}$ is determined by $\Omega_{+}(0)$ and is independent of $\Omega_{-}(0)$. Indeed, if $u$ solves $(2.7)$ with $(2.8)$ then $\theta(u)$ solves $(2.7)$ for continuous nondecreasing $\theta: \mathbb{R} \rightarrow \mathbb{R}$ since $F_{\xi}$ is geometric. Take $\theta(\sigma)=\sigma_{+}=\max (\sigma, 0)$ to observe that $u_{+}=\theta(u)$ solves $(2.7)$. By $(2.8) u_{+}$gives a weak solution $\left\{\Omega_{ \pm}^{\prime}(t)\right\}_{0 \leq t<T}$ with initial data $\left(\Omega_{+}(0), \phi\right)$. By definition of $u_{+}$we see $\Omega_{+}^{\prime}(t)=\Omega_{+}(t)$ and $\Omega_{-}^{\prime}(t)=\phi$. We thus observe that $\Omega_{+}(t)$ is determined by $\Omega_{+}(0)$.

Remark 2.5. The interface $\Gamma(t)$ is defined by the complement of $\Omega_{+}(t) \cup \Omega_{-}(t)$ in $T$. There is a chance that $\Gamma(t)$ develops an interior even if $\Gamma(0)$ is a smooth hypersurface in $\mathbb{T}$. For example consider the equation $V=-1$ and

$$
R=\left\{\left(x_{1}, x_{2}\right) \in \mathbb{R}^{2} ; 0 \leq x_{1}, \leq 2,0 \leq x_{2} \leq 2\right\} .
$$

Suppose that

$$
\Omega_{+}(0)=\left\{x \in \mathbb{T} ; x_{1} \neq 1\right\}, \Omega_{-}(0)=\phi
$$

so that $\Gamma(0)=\left\{x_{1}=1\right\}$. Then $\Omega_{+}(t)=\left\{x \in \mathbf{T} ; 0 \leq x_{1} \leq 2,\left|x_{1}-1\right|>t\right\}$ and $\Omega_{-}(t)=\phi$. Indeed equation (2.7) for this example is

$$
u_{t}+|\nabla u|=0
$$

By definition of viscosity solutins, one can check that

$$
u(t, x)=\left\{\begin{array}{ll}
0 & \text { for }\left|x_{1}-1\right| \leq t \\
x_{1}-1-t & \text { for } x_{1}-1>t \quad\left(x \in \mathbb{R}^{2}\right) \\
1-x_{1}-t & \text { for } x_{1}-1<-t
\end{array} \quad\right.
$$

is a viscosity solution of $(2.14)$ on $(0, \infty) \times \mathbb{T}$. We now observe that $\Omega_{ \pm}(t)$ is given by (2.8) with $u$ of (2.15).

For the mean curvature flow equation

$$
V=-\operatorname{div} \vec{n}
$$

we do not know whether or not $\Gamma(t)$ develops an interior if $\Gamma(0)$ is a smooth hypersurface. As pointed out in [ES1] we know $\Gamma(t)$ may develop an interior if $\Gamma(0)$ has a singularity. 
3. Diffusion equations across interfaces. This section gives a generalized formulation of

$$
\begin{gathered}
v_{t}=\Delta v+g_{ \pm}(v) \quad \text { in } Q_{T}^{ \pm}=\bigcup_{0<t<T}\{t\} \times \Omega_{ \pm}(t), \\
v(t):=v(t, \cdot) \in C^{1}(\mathbb{T}) \quad \text { for } 0<t<T,
\end{gathered}
$$

where

$$
Q_{T}^{ \pm}=\left\{(t, x) \in Q_{T} ; u(t, x) \gtrless 0\right\}, Q_{T}=(0, T) \times \mathbb{T}
$$

with some function $u \in C\left(\bar{Q}_{T}\right)$. The interpretation of the equation on the interface is crucial.

We introduce a multi-valued function $\Phi$ associated with continuous functions $g_{ \pm}(\sigma)$. For $(s, \sigma) \in \mathbb{R}^{2}$ we define a closed interval $\Phi(s, \sigma)$ such that

$$
\Phi(s, \sigma)= \begin{cases}\left\{g_{+}(\sigma)\right\} & \text { if } s>0 \\ {[\underline{g}(\sigma), \bar{g}(\sigma)]} & \text { if } s=0 \\ \left\{g_{-}(\sigma)\right\} & \text { if } s<0\end{cases}
$$

where $\underline{g}(\sigma)=\min \left(g_{+}(\sigma), g_{-}(\sigma)\right), \bar{g}=\max \left(g_{+}, g_{-}\right)$. This correspondence defines a mapping $\Phi: \mathbb{R}^{2} \rightarrow 2^{\mathbb{R}}$. For $u, v \in C\left(\bar{Q}_{T}\right)$ we define a subset $G(u, v)$ such that

$$
G(u, v)=\left\{q \in L^{\infty}\left(Q_{T}\right) ; q(z) \in \Phi(u(z), v(z)) \text { a.e. } z \in Q_{T}\right\},
$$

where $z=(t, x)$. This correspondence defines a mapping $G: C\left(\bar{Q}_{T}\right) \times C\left(\bar{Q}_{T}\right) \rightarrow$ $2^{L^{\infty}\left(Q_{T}\right)}$.

Definition 3.1. Suppose that $u \in C\left(\bar{Q}_{1}\right)$ is given and that $Q_{T}^{ \pm}$is defined by (3.3). Suppose that $g_{ \pm}: \mathbb{R} \rightarrow \mathbb{R}$ is continuous. We say $v \in C\left(\bar{Q}_{T}\right)$ is a generalized solution of $(3.1)-(3.2)$ if

$$
v_{t}-\Delta v \in G(u, v) \text { in } Q_{T}
$$

i.e., there is $q \in G(u, v)$ such that

$$
v_{t}-\Delta v=q \text { in } Q_{T}
$$

in the distribution sense. Since $G(u, v)$ depends on $u$ only through its signature, this definition only depends on $Q_{T}^{ \pm}$and is independent of the choice of $u$.

Proposition 3.2. For $u, v \in C\left(\bar{Q}_{T}\right)$ the set $G(u, v)$ is a nonempty, bounded convex subset of $L^{\infty}\left(Q_{T}\right)$.

Proof. Since $\Phi(s, \sigma)$ is convex in $\mathbb{R}$,

$$
\lambda q_{1}(z)+(1-\lambda) q_{2}(z) \in \Phi(u(z), v(z)) \quad \text { for a.e. } z
$$

if $q_{1}, q_{2} \in G(u, v)$ and $0<\lambda<1$. This implies that $\lambda q_{1}+(1-\lambda) q_{2} \in G(u, v)$ so $G(u, v)$ is convex in $L^{\infty}\left(Q_{T}\right)$.

The Borel measurable function

$$
\psi(s, \sigma)=\chi_{(-\infty, 0)}(s) g_{-}(\sigma)+\chi_{[0, \infty)}(s) g_{+}(\sigma)
$$


on $\mathbb{R}^{n}$ satisfies $\psi(s, \sigma) \in \Phi(s, \sigma)$ for all $s, \sigma \in \mathbb{R}$, and therefore $\psi(u, v) \in G(u, v)$.

Since $g_{ \pm}$is locally bounded, we see $G(u, v)$ is bounded in $L^{\infty}\left(Q_{T}\right)$. $\square$

Lemma 3.3. Suppose that $u_{m} \rightarrow u$ in $C\left(\bar{Q}_{T}\right)$ and that $v_{m} \rightarrow v$ in $C\left(\bar{Q}_{T}\right)$. Suppose that $q_{m} \in G\left(u_{m}, v_{m}\right)$. Then there is a subsequence $\left\{m_{j}\right\}$ and $q \in G(u, v)$ such that $q_{m_{j}} \rightarrow q *-$ weakly in $L^{\infty}\left(Q_{T}\right)$.

Proof. Since $g_{ \pm}$is continuous, $\bigcup_{m=1}^{\infty} G\left(u_{m}, v_{m}\right)$ is bounded in $L^{\infty}\left(Q_{T}\right)$. In particular $\left\{q_{m}\right\}$ is bounded in $L^{\infty}\left(Q_{T}\right)$. By the Banach-Alaoglu theorem $\left\{q_{m}\right\}$ has a *-weak convergent subsequence (still denoted $\left\{q_{m}\right\}$ ), i.e.,

$$
q_{m} \rightarrow q * \text {-weakly in } L^{\infty}\left(Q_{T}\right) .
$$

In particular $q_{m} \rightarrow q$ weakly in $L^{2}\left(Q_{T}\right)$ since $Q_{T}$ is bounded. Applying Mazur's theorem (see e.g. [Y]) we see there is $\lambda_{m}^{m}, \ldots, \lambda_{m}^{\ell m} \geq 0$ with

$$
\sum_{j=m}^{\ell_{m}} \lambda_{m}^{j}=1
$$

such that

$$
\tilde{q}_{m}:=\sum_{j=m}^{\ell_{m}} \lambda_{m}^{j} q_{j} \rightarrow q \text { strongly in } L^{2}\left(Q_{T}\right) \text { as } m \rightarrow \infty .
$$

Taking a subsequence if necessary we may conclude

$$
\tilde{q}_{m}(z) \rightarrow q(z) \quad(m \rightarrow \infty) \text { for a.e. } z \text {. }
$$

We fix $z \in Q_{T}$ such that (3.6) and

$$
q_{m}(z) \in \Phi\left(u_{m}(z), v_{m}(z)\right) \text { for all } m \geq 1 .
$$

Suppose that $u(z)=0$. By $(3.4)$ and (3.7)

$$
q_{m}(z) \in\left[\underline{g}\left(v_{m}(z)\right), \bar{g}\left(v_{m}(z)\right)\right]
$$

since $\left\{g_{ \pm}\left(v_{m}(z)\right)\right\}$ lies in the interval in (3.8). Since $\underline{g}$ and $\bar{g}$ are continuous and $v_{m}(z) \rightarrow v(z)$, for each $\varepsilon>0$ there is $m_{0}$ such that if $m \geq m_{0}$ then

$$
\left[\underline{g}\left(v_{m}(z)\right), \bar{g}\left(v_{m}(z)\right)\right] \subset(a-\varepsilon, b+\varepsilon)
$$

with

$$
[a, b]:=[\underline{g}(v(z)), \bar{g}(v(z))] .
$$

By (3.8) we now observe that

$$
\tilde{q}_{m}(z) \in(a-\varepsilon, b+\varepsilon) .
$$

From (3.6) it follows that

$$
q(z) \in(a-\varepsilon, b+\varepsilon) .
$$

Since $\varepsilon>0$ is arbitrary, this implies

$$
q(z) \in[\underline{g}(v(z)), \bar{g}(v(z))] .
$$


Suppose that $u(z)>0$. For sufficiently large $m$, say $m \geq m_{0}$, we may assume $u_{m}(z)>0$. It follows that

$$
\Phi\left(u_{m}(z), v_{m}(z)\right)=\left\{g_{+}\left(v_{m}(z)\right)\right\} \text { for } m \geq m_{0} .
$$

By (3.7) we have

$$
q_{m}(z)=g_{+}\left(v_{m}(z)\right) \text { for } m \geq m_{0} .
$$

Since $\tilde{q}_{m}(z) \rightarrow q(z)$ by (3.6) and $q_{+}$is continuous, (3.10) yields

$$
q(z)=q_{+}(v(z)) .
$$

The proof for $u(z)<0$ parallels that for $u(z)>0$. By (3.9) and (3.11) one can conclude that

$$
q(z) \in \Phi(u(z), v(z)), \text { a.e. } z \in Q_{T},
$$

which completes the proof.

Corollary 3.4. The set $G(u, v)$ is weak * compact in $L^{\infty}\left(Q_{T}\right)$.

Proof. By Lemma 3.3 we see $G(u, v)$ is weak * sequencially closed. Since $G(u, v)$ is bounded by Proposition 3.2 and since the predual $L^{1}\left(Q_{T}\right)$ is separable, one can drop the word "sequentially". The boundedness of $G(u, v)$ now implies that $G(u, v)$ is weak * compact.

Remark 3.5. The condition (3.2) is implicit in Definition 3.1. We will see that all generalized solution $v$ has the regularity property (3.2).

4. Main results. We consider a system (3.1) - (3.2) coupled with an interface equation:

$$
\begin{aligned}
v_{t} & =\Delta v+g_{ \pm}(v) \quad \text { in } Q_{T}^{ \pm}=\bigcup_{0<t<T}\{t\} \times \Omega_{ \pm}(t) \\
v(t) & =v(t, \cdot) \in C^{1}(\mathbb{T}) \quad \text { for } 0<t<T \\
V & =\eta(\vec{n}, \nabla \vec{n})+W(v) \alpha(\vec{n}) \quad \text { on } \Gamma(t)=\mathbb{T} \backslash\left(\Omega_{+}(t) \cup \Omega_{-}(t)\right)
\end{aligned}
$$

with given initial data

$$
\begin{aligned}
v(0, x) & =v_{0}(x) \text { in } \mathrm{T} \\
\left.\Omega_{ \pm}(t)\right|_{t=0} & =\Omega_{ \pm}(0) .
\end{aligned}
$$

Here we assume that

$$
\begin{aligned}
& g_{ \pm}: \mathbb{R} \rightarrow \mathbb{R} \text { is continuous and bounded } \\
& \eta \text { satisfies }(2.9)-(2.11) \\
& W: \mathbb{R} \rightarrow \mathbb{R} \text { is continuous } \\
& \alpha: S^{n-1} \rightarrow \mathbb{R} \text { is continuous }
\end{aligned}
$$

We say $\left(\Omega_{ \pm}(t), v(t)\right)$ is a weak solution of $(4.1)-(4.5)$ if $\left\{\Omega_{ \pm}(t)\right\}_{0 \leq t<T}$ is a weak solution of (4.3), (4.5) with $v \in C\left(\bar{Q}_{T}\right)$ and $v$ is a generalized solution of (4.1)-(4.2) with (4.4); see Definitions 2.1 and 3.1. We now state one of our main results. 
THEOREM 4.1. Let $T>0$. Assume that $g_{ \pm}, \eta, W, \alpha$ satisfy (4.6a-d). Suppose that $\Omega_{+}(0)$ and $\Omega_{-}(0)$ are mutually disjoint open sets in $\mathrm{T}$ and that $v_{0}(x) \in C^{2}(\mathbb{T})$. Then there exists a (global) weak solution $\left(\Omega_{ \pm}(t), v(t)\right)$ of (4.1)-(4.5) such that $v \in$ $C^{1,0}\left(\bar{Q}_{T}\right)=\left\{v \in C\left(\bar{Q}_{T}\right) ; \nabla v \in C\left(\bar{Q}_{T}\right)\right\}$.

Remark 4.2. We note that (4.3) includes

$$
V=-c \operatorname{div} \vec{n}+W(v), \quad c \geq 0
$$

as a special example. If $\left(4.3^{\prime}\right)$ replaces $(4.3)$ in $(4.1)-(4.5)$, then it is known that there is a unique smooth local solution. This is proved by X.-Y. Chen [XYC] for $c>0$ and by $\mathrm{X}$. Chen [XC2] for $c=0$ where $\mathbb{R}^{n}$ replaces $\mathbf{T}$. Our result is the first global existence result even for this special system if the space dimension $n \geq 2$. For $n=1$ see $[\mathrm{HNM}]$.

We shall construct a solution using Kakutani's fixed point theory for a multivalued mapping. We take a Banach space

$$
X:=C^{1,0}\left(\bar{Q}_{T}\right) .
$$

For $v \in X$ we solve (4.3), (4.5) by applying Theorem 2.2. Since $v$ can be extended continuously for $t>T$ we have a unique weak solution $\left\{\Omega_{ \pm}(t)\right\}_{0 \leq t \leq T}$ for (4.3), (4.5) with given $\Omega_{ \pm}(0)$. If we set

$$
\widetilde{Q}_{T}^{ \pm}=\bigcup_{0 \leq t \leq T}\{t\} \times \Omega_{ \pm}(t)
$$

then we have a mapping

$$
\mathcal{T}: X \rightarrow \mathcal{O}, v \mapsto\left(\widetilde{Q}_{T}^{+}, \widetilde{Q}_{T}^{-}\right),
$$

where $\mathcal{O}$ denotes the set of disjoint pair of open sets in $[0, T] \times \mathbb{T}$.

For $q \in L^{\infty}\left(Q_{T}\right)$ let $w=E(q)$ be the unique solution of

$$
\begin{aligned}
w_{t}-\Delta w & =q \text { in } Q_{T} \\
w(0, x) & =v_{0}(x) \in C^{2}(\mathbb{T}) .
\end{aligned}
$$

By the parabolic theory [LUS] $E$ defines a continuous linear operator from $L^{\infty}\left(Q_{T}\right)$ to $\cap_{p>1} W_{p}^{2,1}\left(Q_{T}\right)$, which is continuously embedded in $X$ by the Sobolev inequality. Thus

$$
E: L^{\infty}\left(Q_{T}\right) \rightarrow X
$$

is a bounded linear operator. For $u, v \in C\left(\bar{Q}_{T}\right)$ we define a subset of $X$ by

$$
\mathcal{P}(u, v)=\{E(q) ; q \in G(u, v)\} .
$$

This correspondence defines a mapping

$$
\mathcal{P}: C\left(\bar{Q}_{T}\right) \times C\left(\bar{Q}_{T}\right) \rightarrow 2^{X} .
$$

For given $\left(\widetilde{Q}_{T}^{+}, \widetilde{Q}_{T}^{-}\right) \in \mathcal{O}$ we take $u \in C\left(\bar{Q}_{T}\right)$ such that

$$
\widetilde{Q}_{T}^{ \pm}=\left\{(t, x) \in \bar{Q}_{T} ; u \gtrless 0\right\} .
$$


Since $G$ depends on $u$ through its signature, one may regard the mapping $\mathcal{P}$ as

$$
\mathcal{P}: \mathcal{O} \times C\left(\bar{Q}_{T}\right) \rightarrow 2^{X} .
$$

For given $v_{0}$ and $\Omega_{ \pm}(0)$ we define

$$
\mathcal{S}: X \rightarrow 2^{X}
$$

by $\mathfrak{S}(v)=\mathcal{P}(\mathcal{T}(v), v)$. If $\mathcal{S}$ has a fixed point $\bar{v} \in X$, i.e.,

$$
\bar{v} \in S(\bar{v})
$$

it is easy to see that $\left(\Omega_{ \pm}(t), \bar{v}(t)\right)$ is a weak solution of $(4.1)-(4.5)$ where

$$
\mathcal{T}(\bar{v})=\bigcup_{0 \leq t \leq T}\{t\} \times \Omega_{ \pm}(t) .
$$

We shall prove that $\mathcal{S}$ has a fixed point.

Proposition 4.3. The set $\mathcal{P}(u, v)$ is nonempty, compact and convex in $C\left(\bar{Q}_{T}\right)$ (and in $X$ ).

Proof. Since $E$ defined by (4.7) is linear and $G(u, v)$ is nonempty and convex by Proposition 3.2 we see $\mathcal{P}(u, v)$ is convex.

We next observe that $E$ is continuous from a bounded set of $L^{\infty}\left(\bar{Q}_{T}\right)$ (equipped with weak * topology) to $X$. Indeed, if $q_{m} \rightarrow q *$ weakly in $L^{\infty}\left(Q_{T}\right)$ then $\left\{E\left(q_{m}\right)\right\}$ has a weakly convergent subsequence in $W_{p}^{2,1}\left(Q_{T}\right)$ for $p>1$. Since the inclusion

$$
W_{p}^{2,1}\left(Q_{T}\right) \rightarrow X=C^{1,0}\left(\bar{Q}_{T}\right) \text { is compact if } p>n+1
$$

(see e.g. [LUS]), $E\left(q_{m}\right) \rightarrow w$ strongly in $X$ by taking a subsequence. Since $w_{m}=$ $E\left(q_{m}\right)$ satisfies

$$
\left(\partial_{t}-\Delta\right) w_{m}=q_{m} \text { in } Q_{T}, \quad w_{m}(0, x)=v_{0}(x),
$$

$w$ solves

$$
\left(\partial_{t}-\Delta\right) w=q \text { in } Q_{T}
$$

in the distribution sense with $w(0, x)=v_{0}(x)$. This implies $w=E(q)$. Since the limit $w$ is independent of the choice of subsequences, we observe

$$
E\left(q_{m}\right) \rightarrow E(q) \text { in } X
$$

This sequential continuity implies the continuity on a bounded set of $L^{\infty}\left(Q_{T}\right)$.

Since $G(u, v)$ is weak * compact in $L^{\infty}\left(Q_{T}\right)$, the continuous image of $G(u, v)$ is compact. The above continuity of $E$ implies that $\mathcal{P}(u, v)$ is compact in $C^{0}\left(\bar{Q}_{T}\right)$ as well as in $X$. $\square$

Since $g_{ \pm}$is bounded by (4.6a), we see

$$
\bigcup_{u, v \in C\left(\bar{Q}_{T}\right)} G(u, v)
$$

is bounded in $L^{\infty}\left(Q_{T}\right)$. Therefore, by the parabolic theory for (4.7) [LUS]

$$
\mathcal{S}(v) \subset K=\left\{w \in W_{p}^{2,1}\left(Q_{T}\right) ;\|w\|_{W_{p}^{2,1}} \leq M\right\}, p>1
$$


if $M$ is taken sufficiently large. We fix $p>n+1$ so that $K$ is compact in $X$ by (4.8). The mapping $\mathcal{S}$ is now interpreted as

$$
\mathcal{S}: X \rightarrow 2^{K}
$$

The graph of $\mathcal{S}$ is defined by

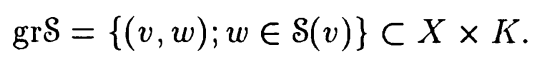

Since $K$ is compact, grS is closed if and only if $\mathcal{S}$ is upper semicontinuous. For the definition of upper semicontinuity see [AF].

Proposition 4.4. The set $\mathrm{grS}$ is closed in $X \times K$.

Proof. Suppose that $v_{m}, v \in X, w_{m} \in \mathcal{S}\left(v_{n}\right), w \in X$ such that $v_{m} \rightarrow v$ in $X$ and $w_{m} \rightarrow w$ in $X$. Our goal is to prove $w \in \mathcal{S}(v)$. By the definition of weak solutions for (4.3) there is a viscosity solution $u_{m} \in C\left(\bar{Q}_{T}\right)$ of

$$
u_{t}+F_{m}\left(t, x, \nabla u, \nabla^{2} u\right)=0 \text { in } Q_{T}
$$

with

$$
F_{m}(t, x, p, X)=F_{\eta}(p, X)-W\left(v_{m}(t, x)\right) \alpha(-p /|p|)|p|
$$

such that

$$
\mathcal{T}\left(v_{m}\right)=\left(\left\{u_{m}(t, x)>0\right\},\left\{u_{m}(t, x)<0\right\}\right) .
$$

One can arrange $u_{m}(0, x)=u_{0}(x)$ independent of $m$ such that

$$
\Omega_{ \pm}(0)=\left\{x \in \mathbb{T} ; u_{0}(x) \gtrless 0\right\} .
$$

Since $v_{m} \rightarrow v$ in $C\left(\bar{Q}_{T}\right)$, by the stability of viscosity solutions there is $u \in C\left(\bar{Q}_{T}\right)$ such that $u_{m} \rightarrow u$ in $C\left(\bar{Q}_{T}\right)$ and $u$ solves (in the viscosity sense)

$$
u_{t}+F\left(t, x, \nabla u, \nabla^{2} u\right)=0 \text { in } Q_{T}
$$

with $F(t, x, p, X)=F_{\eta}(p, X)-W(v(t, x)) \alpha(-p /|p|)|p|$ (see Theorem in Appendix), where $u(0, x)=u_{0}(x)$. This implies

$$
\mathcal{T}(v)=(\{u>0\},\{u<0\}) .
$$

By the definition of $\mathcal{P}$ there is $q_{m} \in G\left(u_{m}, v_{m}\right)$ such that

$$
\begin{aligned}
\left(\partial_{t}-\Delta\right) w_{m} & =q_{m} \text { in } Q_{T}, \\
w_{m}(0, x) & =v_{0}(x) \text { on } \mathbb{T} .
\end{aligned}
$$

Applying Lemma 3.3, we may conclude that

$$
q_{m} \rightarrow q * \text {-weakly in } L^{\infty}\left(Q_{T}\right)
$$

with some $q \in G(u, v)$ by taking a subsequence if necessary. Since $w_{m} \rightarrow w$ in $X$, (4.10) implies that

$$
\left(\partial_{t}-\Delta\right) w=q \text { in } Q_{T}
$$


in the distribution sense and

$$
w(0, x)=v_{0}(x) .
$$

This yields $w \in S(v)$ by (4.9) so the proof is now complete.

Proof of Theorem 4.1. Since $K$ is compact and convex by Propositions 4.3 and 4.4 one can apply the following fixed point theorem to conclude that there is $\bar{v} \in \mathcal{S}(\bar{v}) \cap K$. By the definition of $\mathcal{S}$ we see $\bar{v}$ together with $\mathcal{T}(\bar{v})$ is a desired weak solution of (4.1)(4.5).

Kakutani's Fixed Point theorem [AF, Theorem 3.2.3]. Let $K$ be a convex compact subset of a Banach space $X$ and $\mathcal{S}: X \rightarrow 2^{K}$. If $\mathcal{S}$ is upper semicontinuous and $\mathcal{S}(v)$ is a convex closed set in $K$ for $v \in X$, then $\mathcal{S}$ has a fixed point $\bar{v} \in K \cap \mathcal{S}(\bar{v})$.

Remark 4.5. The assumption $v_{0}(x) \in C^{2}(T)$ in Theorem 4.1 is weakened as $v_{0}(x) \in W^{2-2 / p}(\mathbb{T}), p>n+1$ because the regularity condition on $v_{0}$ is only used to solve (4.7) in $W_{p}^{2,1}\left(\bar{Q}_{T}\right)$.

We conclude this paper by stating an existence result of a global solution on the time interval $(0, \infty)$.

THEOREM 4.6. Assume the same hypotheses of Theorem 4.1 for $g_{t}, \eta, w, \alpha, \Omega_{ \pm}(0)$. Suppose that $v_{0} \in W^{2-2 / p}(\mathbb{T})$ for $p>n+1$. Then there exists $\left\{\left(\Omega_{ \pm}(t), v(t)\right)\right\}_{t \geq 0}$ which is a weak solution of (4.1) - (4.5) for arbitrary $T>0$. implies

Proof. For fixed $T>0$ by Remark 4.5 there is $v_{T}$ such that $v_{T} \in \mathcal{S}\left(v_{T}\right)$. This

$$
\begin{aligned}
\left(\partial_{t}-\Delta\right) v_{T} & =q_{T} \text { in } Q_{T}, \\
v_{T}(0, x) & =v_{0}(x),
\end{aligned}
$$

with

$$
q_{T} \in \bigcup_{u, v \in C\left(\bar{Q}_{T}\right)} G(u, v)
$$

Since $g_{ \pm}$is bounded by (4.6a) we observe

$$
\left|q_{T}\right|_{L^{\infty}\left(Q_{T}\right)} \leq M=\sup _{\sigma}\left|g_{ \pm}(\sigma)\right|
$$

By the parabolic regularity theory [LUS] $\left\{v_{T}\right\}_{T \geq 1}$ is bounded in $W_{p}^{2,1}\left(\bar{Q}_{t_{0}}\right)(p>n+1)$ for each $t_{0}>0$. By (4.8) and a diagonal argument there is a subsequence $\left\{v_{T^{\prime}}\right\}$ and $v \in C([0, \infty) \times \mathbb{T})$ such that

$$
v_{T^{\prime}} \rightarrow v \text { in } X_{t_{0}}=C^{1,0}\left(\bar{Q}_{t_{0}}\right)
$$

Since $v_{T^{\prime}} \in \mathcal{S}\left(v_{T^{\prime}}\right) \subset X_{t_{0}}$ and grS is closed, (4.11) implies $v \in \mathcal{S}(v) \subset X_{t_{0}}$ where $\mathcal{S}$ depends on $t_{0}$. Since $t_{0}$ is arbitrary, this yields a desired global solution on $[0, \infty)$. $\square$

Appendix. We shall state stability properties of viscosity solutions used in the proof of Proposition 4.4 for the reader's convenience. We use a following notation. For $h_{m}: L \rightarrow \mathbb{R}, L \subset Z$ we define

$$
\begin{aligned}
& \lim _{*} h_{m}: \bar{L} \rightarrow \mathbb{R} \cup\{-\infty\} \\
& \lim _{m}^{*} h_{m}: \bar{L} \rightarrow \mathbb{R} \cup\{+\infty\}
\end{aligned}
$$


by

$$
\begin{aligned}
& \left(\lim _{*} h_{m}\right)(z)=\lim _{\substack{m \rightarrow \infty \\
\varepsilon \downarrow 0}} \inf \left\{h_{j}(y), d(z, y)<\varepsilon, j \geq m, y \in L\right\} \\
& \text { and } \lim ^{*} h_{m}=-\lim _{*}\left(-h_{m}\right)
\end{aligned}
$$

where $Z$ is a metric space with the metric $d$. If $h$ is independent of $m$, we write $h_{*}=\lim _{*} h_{m}, h^{*}=\lim ^{*} h_{m}$. We shall suppress the word "viscosity".

Lemma. Suppose that $F_{m}: Q_{T} \times \mathbb{R}^{n} \times \mathbb{S}_{n} \rightarrow \mathbb{R}$ is lower semicontinuous and that $F=\lim _{*} F_{m}$. Suppose that $u_{m}$ is a subsolution of

$$
u_{t}+F_{m}\left(t, x, \nabla u, \nabla^{2} u\right)=0 \text { in } Q_{T} .
$$

Then $u=\lim ^{*} u_{m}$ is a subsolution of

$$
u_{t}+F\left(t, x, \nabla u, \nabla^{2} u\right)=0 \text { in } Q_{T}
$$

provided that $u$ does not take $+\infty$ in $Q_{T}$.

Similar results are proved by Barles and Perthame [BP] for first order differential equations and formulated in Ishii [I] in the general case. Since the proof is easily modified for our setting, we omit the proof. The following is a simple application of Lemma, the comparison Proposition 2.3, and construction of sub and supersolutions.

THEOREM. Suppose that $\eta, W, \alpha$ satisfy $\left(\begin{array}{lll}4.6 & b-d\end{array}\right)$. Suppose that $v_{m} \rightarrow v$ in $C\left(\bar{Q}_{T}\right)$. We set

$$
\begin{aligned}
F_{m} & =F_{\eta}(p, X)-W\left(v_{m}(t, x)\right) \alpha(-p /|p|)|p| \\
F & =F_{\eta}(p, X)-W(v(t, x)) \alpha(-p /|p|)|p|
\end{aligned}
$$

where $F_{\eta}$ is defined by (2.3). Suppose that $u_{m} \in C\left(\bar{Q}_{T}\right)$ is a solution of

$$
u_{t}+F_{m}\left(t, x, \nabla u, \nabla^{2} u\right)=0 \text { in } Q_{T}
$$

with $u_{m}(0, x)=u_{0}(x) \in C(\mathbb{T})$. Then $u_{m} \rightarrow u$ in $C\left(\bar{Q}_{T}\right)$ for some $u \in C\left(\bar{Q}_{T}\right)$ and $u$ is a solution of

$$
u_{t}+F\left(t, x, \nabla u, \nabla^{2} u\right)=0 \text { in } Q_{T}
$$

with $u(0, x)=u_{0}(x)$.

Proof. Since $v_{m} \rightarrow v$ in $C\left(\bar{Q}_{T}\right)$ there are sub and supersolutions $w_{ \pm}$of (1) such that

$$
\begin{aligned}
& w_{ \pm}(0, x)=u_{0}(x) \\
& w_{-}(t, x) \leq u_{0}(x) \leq w_{+}(t, x) \text { in } Q_{T}
\end{aligned}
$$

and that $w_{ \pm}$is independent of $m$; see [CGG, Proposition 6.4] and the proof of Theorem 2.2. By Proposition 2.3 we see

$$
w_{-} \leq u_{m} \leq w_{+} \text {in } Q_{T} .
$$


Since $u_{m}$ is a subsolution of

$$
u_{t}+\left(F_{m}\right)_{*}\left(t, x, \nabla u, \nabla^{2} u\right)=0 \text { in } Q_{T}
$$

by definition, applying Lemma yields that $\bar{u}=\lim ^{*} u_{m}$ is a subsolution of

$$
u_{t}+F_{*}\left(t, x, \nabla u, \nabla^{2} u\right)=0 \text { in } Q_{T} .
$$

(This is the definition that $\bar{u}$ is a subsolution of (2)). Similarly $\underline{u}=\lim _{*} u_{m}$ is a supersolution of

$$
u_{t}+F^{*}\left(t, x, \nabla u, \nabla^{2} u\right)=0 \text { in } Q_{T} .
$$

By (3) we observe that

$$
\bar{u}(0, x)=\underline{u}(0, x)=u_{0}(x) .
$$

Applying Proposition 2.3 implies $\bar{u}=\underline{u}$ and $u=\bar{u}$ is a solution of (2). The property $\bar{u}=\underline{u}$ implies that $u_{m} \rightarrow u$ in $C\left(\bar{Q}_{T}\right)$. The proof is now complete.

Acknowledgement. This work of the first author was completed while he visited the Institute for Mathematics and its Applications. Its hospitality and support are gratefully acknowledged.

\section{REFERENCES}

[AW] D.G. ARONSON AND H.F. WeINBERGER, Multidimensional nonlinear diffusion arising in population genetics, Adv. in Math., 30 (1978), pp. 33-76.

[AF] J.P. Aubin and H. Frankowska, Set-valued Analysis, Birkhäuser, Boston-Basel-Berlin (1990).

[B] G. BARLES, Remarks on a flame propagation model, Rapport INRIA, \# 464 (1985).

[BP] G. Barles and B. Perthame, Discontinuous solutions of deterministic optimal stopping time problems, RAIRO Modèl. Math. et Anal. Num. 21 (1987), pp. 557-579.

[BK] L. BRONSARD AND R.V. KOHN, Motion by mean curvature as the singular limit of Ginzburg-Landau dynamics, preprint 1989.

[C] G. CaginalP, The role of microscopic anisotropy in the macroscopic behavior of a phase boundary, Annals of Physics, 172 (1986), pp. 136-155.

[CP] J. CARR AND R.L. PEgo, Metastable patterns in solutions of $u_{t}=\varepsilon^{2} u_{x x}-f(u)$, Comm. Pure Appl. Math. 42 (1989), pp. 523-576.

[XC1] X. CHEN, Generation and propagation of the interface for reaction-diffusion equations, IMA preprint \# 637 (1990).

[XC2] X. CHEN, General and propagation of interfaces in reaction-diffusion systems, IMA preprint \# 708 (1990).

[XYC] X.-Y. CHEN, Dynamics of interfaces in reaction diffusion systems, Hiroshima Math. J. 21, to appear (1991).

[CGG] Y.-G. Chen, Y. GIGA AND S. Goto, Uniqueness and existence of viscosity solutions of generalized mean curvature flow equations, J. Differential Geometry 33, to appear (1991); Announcement: Proc. Japan Acad. Ser. A, 65 (1989), pp. 207-210.

[DS] P. DeMottoni and M. Schatzman, Geometrical evolution of developed interfaces, preprint (1990); Announcement: Evolution géometric d'interfaces, C.R. Acad. Sci. Paris, 309, (1989), pp. $453-458$.

[ESS] L.C. Evans, H.M. Soner AND P.E. Songanidis, The Allen-Cahn equation and generalized motion by mean curvature, manuscript.

[ES1] L.C. Evans AND J. SpRUCK, Motion of level sets by mean curvature I, J. Differential Geometry, to appear.

[ES2] L.C. Evans AND J. SPRUCK, Motion of level sets by mean curvature II, J. Differential Geometry, to appear.

[F] P.C. FIFE, Dynamics of Interfacial Layers and Diffusive Interfaces, CBMS-NSF Regional Conference Series in Applied Mathematics 53, SIAM, Philadelphia (1982). 
[FH] P.C. Fife AND L. HsiaO, The generation and propagation of internal layers, Nonlinear Anal. TMA 12 (1988), pp. 19-41.

[GG1] Y. Giga AND S. Goto, Motion of hypersurfaces and geometric equations, Hokkaido University, Preprint Series \# 70 (1990).

[GG2] Y. GIGA AND S. GOTO, Geometric evolution of phase-boundaries, IMA \# 738 (1990).

[GGIS] Y. Giga, S. Goto, H. IshII AND M.-H. SATO, Comparison principle and convexity preserving properties for singular degenerate parabolic equations on unbounded domains, Indiana Univ. Math. J. to appear.

[Gr] M. Grayson, A short note on the evolution of a surface by its mean curvature, Duke Math. J. 58 (1989), pp. 555-558.

[Gu1] M. GuRTIN, Towards a nonequilibrium thermodynamics of two-phase materials, Arch. Rational Mech. Anal. 100 (1988), pp. 275-312.

[Gu2] M. GuRTIN, Multiphase thermomechanics with interfacial structure, 1 . Heat conduction and the capillary balance law, Arch. Rational Mech. Anal. 104 (1988), pp. 195-221.

[HNM] D. Hilhorst, Y. Nishiura AND M. Mimura, A free boundary problem arising from some reaction-diffusion system, preprint.

[I] H. IsHII, A boundary value problem of the Dirichlet type for Hamilton-Jacobi equations, Ann. Sc. Norm. Sup. Pisa, (IV) 16 (1989), pp. 105-135.

[LUS] O. LadYZhenskaya, V. SOlONNIKov and N. URAL'CEVA, Linear and Quasilinear Equations of Parabolic Type, Translations of Mathematical Monographs, vol. 23, AMS (1968).

[OMK] T. Ohta, M. Mimura and R. Kobayashi, Higher dimensional localized patterns in excitable media, Physica D34 (1989), pp. 115-144.

[S] H.M. SONER, Motion of a set by the curvature of its boundary, preprint 1990.

[Y] K. Yosida, Functional Analysis, Springer, Berlin-Heidelberg-New York, 4th edition (1974). 
Ian M. Anderson, Niky Kamran and Peter J. Olver, Internal, external and generalized symmetries

C. Foias and J.C. Saut, Asymptotic integration of Navier-Stokes equations with potential forces. I

Ling Ma, The convergence of semidiscrete methods for a system of reaction-diffusion equations

Adelina Georgescu, Models of asymptotic approximation

A. Makagon and H. Salehi, On bounded and harmonizable solutions on infinite order arma systems

San-Yih Lin and Yan-Shin Chin, An upwind finite-volume scheme with a triangular mesh for conservation laws J.M. Ball, P.J. Holmes, R.D. James, R.L. Pego \& P.J. Swart, On the dynamics of fine structure KangPing Chen and Daniel D. Joseph, Lubrication theory and long waves

J.L. Ericksen, Local bifurcation theory for thermoelastic Bravais lattices

Mario Taboada and Yuncheng You, Some stability results for perturbed semilinear parabolic equations

A.J. Lawrance, Local and deletion influence

Bogdan Vernescu, Convergence results for the homogenization of flow in fractured porous media

Xinfu Chen and Avner Friedman, Mathematical modeling of semiconductor lasers

Yongzhi Xu, Scattering of acoustic wave by obstacle in stratified medium

Songmu Zheng, Global existence for a thermodynamically consistent model of phase field type

Heinrich Freistühler and E. Bruce Pitman, A numerical study of a rotationally degenerate hyperbolic system part I: the Riemann problem

Epifanio G. Virga, New variational problems in the statics of liquid crystals

Yoshikazu Giga and Shun'ichi Goto, Geometric evolution of phase-boundaries

Ling Ma, Large time study of finite element methods for 2D Navier-Stokes equations

Mitchell Luskin and Ling Ma, Analysis of the finite element approximation of microstructure in micromagnetics

M. Chipot, Numerical analysis of oscillations in nonconvex problems

J. Carrillo and M. Chipot, The dam problem with leaky boundary conditions

Eduard Harabetian and Robert Pego, Efficient hybrid shock capturing schemes

B.L.J. Braaksma, Multisummability and Stokes multipliers of linear meromorphic differential equations

Tae Il Jeon and Tze-Chien Sun, A central limit theorem for non-linear vector functionals of vector Gaussian processes

Chris Grant, Solutions to evolution equations with near-equilibrium initial values

Mario Taboada and Yuncheng You, Invariant manifolds for retarded semilinear wave equations

Peter Rejto and Mario Taboada, Unique solvability of nonlinear Volterra equations in weighted spaces

Hi Jun Choe, Holder regularity for the gradient of solutions of certain singular parabolic equations

Jack D. Dockery, Existence of standing pulse solutions for an excitable activator-inhibitory system

Jack D. Dockery and Roger Lui, Existence of travelling wave solutions for a bistable evolutionary ecology model

Giovanni Alberti, Luigi Ambrosio and Giuseppe Buttazzo, Singular perturbation problems with a compact support semilinear term

Emad A. Fatemi, Numerical schemes for constrained minimization problems

Y. Kuang and H.L. Smith, Slowly oscillating periodic solutions of autonomous state-dependent delay equations

Emad A. Fatemi, A new splitting method for scaler conservation laws with stiff source terms

Hi Jun Choe, A regularity theory for a more general class of quasilinear parabolic partial differential equations and variational inequalities

Haitao Fan, A vanishing viscosity approach on the dynamics of phase transitions in Van Der Waals fluids

T.A. Osborn and F.H. Molzahn, The Wigner-Weyl transform on tori and connected graph propagator representations

Avner Friedman and Bei $\mathbf{H u}$, A free boundary problem arising in superconductor modeling

Avner Friedman and Wenxiong Liu, An augmented drift-diffusion model in semiconductor device

Avner Friedman and Miguel A. Herrero, Extinction and positivity for a system of semilinear parabolic variational inequalities

David Dobson and Avner Friedman, The time-harmonic Maxwell equations in a doubly periodic structure

Hi Jun Choe, Interior behaviour of minimizers for certain functionals with nonstandard growth

Vincenzo M. Tortorelli and Epifanio G. Virga, Axis-symmetric boundary-value problems for nematic liquid crystals with variable degree of orientation

Nikan B. Firoozye and Robert V. Kohn, Geometric parameters and the relaxation of multiwell energies

Haitao Fan and Marshall Slemrod, The Riemann problem for systems of conservation laws of mixed type

Joseph D. Fehribach, Analysis and application of a continuation method for a self-similar coupled Stefan system

C. Foias, M.S. Jolly, I.G. Kevrekidis and E.S. Titi, Dissipativity of numerical schemes

D.D. Joseph, T.Y.J. Liao and J.-C. Saut, Kelvin-Helmholtz mechanism for side branching in the displacement of light with heavy fluid under gravity 
Chris Grant, Solutions to evolution equations with near-equilibrium initial values

B. Cockburn, F. Coquel, Ph. LeFloch and C.W. Shu, Convergence of finite volume methods

N.G. Lloyd and J.M. Pearson, Computing centre conditions for certain cubic systems

João Palhoto Matos, Young measures and the absence of fine microstructures in the $\alpha-\beta$ quartz phase transition

L.A. Peletier \& W.C. Troy, Self-similar solutions for infiltration of dopant into semiconductors

H. Scott Dumas and James A. Ellison, Nekhoroshev's theorem, ergodicity, and the motion of energetic charged particles in crystals

Stathis Filippas and Robert V. Kohn, Refined asymptotics for the blowup of $u_{t}-\Delta u=u^{p}$.

Patricia Bauman, Nicholas C. Owen and Daniel Phillips, Maximum principles and a priori estimates for an incompressible material in nonlinear elasticity

Patricia Bauman, Nicholas C. Owen and Daniel Phillips, Maximal smoothness of solutions to certain Euler-Lagrange equations from nonlinear elasticity

Jack Carr and Robert Pego, Self-similarity in a coarsening model in one dimension

J.M. Greenberg, The shock generation problem for a discrete gas with short range repulsive forces

George R. Sell and Mario Taboada, Local dissipativity and attractors for the Kuramoto-Sivashinsky equation in thin $2 \mathrm{D}$ domains

T. Subba Rao, Analysis of nonlinear time series (and chaos) by bispectral methods

Nicholas Baumann, Daniel D. Joseph, Paul Mohr and Yuriko Renardy, Vortex rings of one fluid in another free fall

Oscar Bruno, Avner Friedman and Fernando Reitich, Asymptotic behavior for a coalescence problem

Johannes C.C. Nitsche, Periodic surfaces which are extremal for energy functionals containing curvature functions

F. Abergel and J.L. Bona, A mathematical theory for viscous, free-surface flows over a perturbed plane Gunduz Caginalp and Xinfu Chen, Phase field equations in the singular limit of sharp interface problems

Robert P. Gilbert and Yongzhi Xu, An inverse problem for harmonic acoustics in stratified oceans

Roger Fosdick and Eric Volkmann, Normality and convexity of the yield surface in nonlinear plasticity

H.S. Brown, I.G. Kevrekidis and M.S. Jolly, A minimal model for spatio-temporal patterns in thin film flow

Chao-Nien Chen, On the uniqueness of solutions of some second order differential equations

Xinfu Chen and Avner Friedman, The thermistor problem for conductivity which vanishes at large temperature

Xinfu Chen and Avner Friedman, The thermistor problem with one-zero conductivity

E.G. Kalnins and W. Miller, Jr., Separation of variables for the Dirac equation in Kerr Newman space time

E. Knobloch, M.R.E. Proctor and N.O. Weiss, Finite-dimensional description of doubly diffusive convection

V.V. Pukhnachov, Mathematical model of natural convection under low gravity

M.C. Knaap, Existence and non-existence for quasi-linear elliptic equations with the p-laplacian involving critical Sobolev exponents

Stathis Filippas and Wenxiong Liu, On the blowup of multidimensional semilinear heat equations

A.M. Meirmanov, The Stefan problem with surface tension in the three dimensional case with spherical symmetry: non-existence of the classical solution

Bo Guan and Joel Spruck, Interior gradient estimates for solutions of prescribed curvature equations of of parabolic type

Hi Jun Choe, Regularity for solutions of nonlinear variational inequalities with gradient constraints

Peter Shi and Yongzhi Xu, Quasistatic linear thermoelasticity on the unit disk

Satyanad Kichenassamy and Peter J. Olver, Existence and non-existence of solitary wave solutions to higher order model evolution equations

Dening Li, Regularity of solutions for a two-phase degenerate Stefan Problem

Marek Fila, Bernhard Kawohl and Howard A. Levine, Quenching for quasilinear equations

Yoshikazu Giga, Shun'ichi Goto and Hitoshi Ishii, Global existence of weak solutions for interface equations coupled with diffusion equations

Mark J. Friedman and Eusebius J. Doedel, Computational methods for global analysis of homoclinic and hetero clinic orbits: a case study

Mark J. Friedman, Numerical analysis and accurate computation of heteroclinic orbits in the case of center manifolds

809 Peter W. Bates and Songmu Zheng, Inertial manifolds and inertial sets for the phase-field equations

810 J. López Gómez, V. Márquez and N. Wolanski, Global behavior of positive solutions to a semilinear equation with a nonlinear flux condition

811 Xinfu Chen and Fahuai Yi, Regularity of the free boundary of a continuous casting problem

812 Eden, A., Foias, C., Nicolaenko, B. and Temam, R., Inertial sets for dissipative evolution equations Part I: Construction and applications

813 Jose-Francisco Rodrigues and Boris Zaltzman, On classical solutions of the two-phase steady-state Stefan problem in strips

814 Viorel Barbu and Srdjan Stojanovic, Controlling the free boundary of elliptic variational inequalities on a variable domain 